\title{
Percepciones de riesgo al covid-19 entre jóvenes de comunidades indígenas y rurales del sureste de México
}

\author{
Dr. Sergio Meneses-Navarro \\ sergio.meneses@insp.mx \\ CONACYT - Centro de Investigación en Sistemas de Salud, INSP, \\ México \\ Dra. Flor M. Bermúdez-Urbina \\ fmarber79@gmail.com \\ Instituto de Investigaciones en Educación, Universidad Veracruzana, \\ México \\ Dra. Nancy L. Hernández-Reyes \\ nancylet54@hotmail.com \\ Universidad Autónoma de Chiapas, \\ México \\ Dra. N. Gabriela Santos-Hernández \\ nancy.santos@unicach.mx \\ Instituto de Ciencias Biológicas, Universidad de Ciencias y Artes de Chiapas, \\ México \\ Dr. José A. De Fuentes-Vicente \\ jose.defuentes@unicach.mx \\ Instituto de Ciencias Biológicas, Universidad de Ciencias y Artes de Chiapas, \\ México
}

\section{RESUMEN}

Introducción. El 30 de enero de 2020, la OMS declaró emergencia sanitaria por la aparición de un nuevo coronavirus: SARS-CoV-2, que provoca la enfermedad COVID19. En las primeras fases de una pandemia, adoptar conductas de precaución es crucial para prevenir una rápida propagación de la enfermedad. Objetivo. Analizar las percepciones de riesgo, emociones y comportamientos de jóvenes estudiantes residentes de regiones rurales e indígenas de gran marginación de Chiapas, México, sobre el COVID-19. Metódos. Se aplicó una encuesta a jóvenes estudiantes del Colegio de Bachilleres de Chiapas, ubicados en 17 municipios indígenas y rurales de alta y muy alta marginación. Resultados. 971 jóvenes respondieron la encuesta; $47.6 \%$ fueron hablantes de alguna lengua indígena. 92.2\% señaló conocer o haber escuchado sobre 
COVID-19, el $88.4 \%$ cree en su existencia y el $76.4 \%$ señaló sentirse amenazado o muy amenazado. El $39.2 \%$ cree que el virus fue creado en un laboratorio y el $26.9 \%$ opina que fue hecho por el gobierno. Conclusiones. Es necesario implementar estrategias de promoción y educación para la salud con enfoque intercultural que contribuya a contrarrestar la desinformación y generar confianza en las autoridades y la respuesta sanitaria para hacer frente a la pandemia.

Palabras clave: covid-19; poblaciones indígenas; jóvenes 


\title{
Perceptions of risk to covid-19 among young people from indigenous and rural communities in southeast Mexico
}

\begin{abstract}
Introduction. On January 30, 2020, the WHO declared a health emergency due to the appearance of a new coronavirus: SARS-CoV-2, which causes the COVID-19 disease. In the early stages of a pandemic, adopting precautionary behavior is crucial to preventing the rapid spread of the disease. Objective. Analyze the perceptions of risk, emotions and behaviors of young students residing in rural and indigenous regions of great marginalization of Chiapas, Mexico, on COVID-19. Methods. A survey was applied to young students of the Colegio de Bachilleres de Chiapas, located in 17 indigenous and rural municipalities with high and very high marginalization. Results. 971 young people answered the survey; $47.6 \%$ were speakers of an indigenous language. 92.2\% indicated knowing or having heard about COVID-19, 88.4\% believe in its existence and $76.4 \%$ indicated feeling threatened or very threatened. $39.2 \%$ believe that the virus was created in a laboratory and $26.9 \%$ believe that it was made by the government. Conclusions. It is necessary to implement health promotion and education strategies with an intercultural approach that helps to counteract misinformation and build trust in the authorities and the health response to face the pandemic.
\end{abstract}

Keywords: covid-19; indigenous populations; youths

Artículo recibido: 02 noviembre. 2021 Aceptado para publicación: 28 noviembre 2021 Correspondencia: jose.defuentes@unicach.mx Conflictos de Interés: Ninguna que declarar 


\section{INTRODUCCIÓN}

La pandemia del coronavirus SARS-CoV-2, causante de la enfermedad COVID-19, se extendió rápidamente por el mundo desde su aparición en Wuhan, China, a finales del 2019. México ha sido uno de los países más afectados (Sánchez-Talanquer et al., 2001); el 27 de febrero de 2020 se notificó el primer caso y el 18 de marzo la primera defunción por COVID-19 en el país (Suárez et al., 2020). Desde entonces, la enfermedad se ha propagado en todo el territorio nacional, incluyendo las comunidades indígenas y rurales (Ibarra et al., 2021).

Al inicio, la respuesta del sistema de salud se centró en las actividades de contención y mitigación en áreas urbanas ya que, en las primeras fases de una potencia pandemia, adoptar comportamientos de precaución es a menudo el único medio para prevenir una propagación rápida. Sin embargo, la respuesta sanitaria en las regiones rurales e indígenas del país fue menos oportuna y omitió implementar acciones específicas para afrontar tres desafíos principales (Meneses-Navarro et al., 2020):

1. La enraizada desconfianza e incredulidad hacia las autoridades, que incluso llegó a expresarse en formas de rechazo y violencia hacia el personal de salud, señalándolos como fuente de contagio (De Fuentes-Vicente et al., 2021; Haro, 2020; Miranda, 2020);

2. La desinformación, la proliferación de rumores e información falsa y el pensamiento mágico como explicación de la pandemia (De Fuentes-Vicente et al., 2021);

3. La marginación estructural que hace más difícil el acceso de estas poblaciones a los bienes y servicios básicos para afrontar y prevenir la infección, así como la información sanitaria, el agua, el jabón y los cubrebocas (Pelcastre-Villafuerte et al., 2020).

Aunque la mayor carga de la enfermedad se concentra en las principales urbes, la letalidad ha sido mayor entre las poblaciones indígenas que son tratadas principalmente de manera ambulatoria (Argoty-Pantoja et al., 2021). Cifras oficiales señalan que la tasa de letalidad es dos veces mayor para la población que habla una lengua indígena, con un $18.3 \%$ contra 9\%, en febrero de 2021(Sánchez-Talanquer et al., 2021). Aunque se reconocen las desigualdades estructurales en salud entre las poblaciones indígenas y rurales (Pelcastre-Villafuerte et al., 2020), no contamos con estudios específicos que contribuyan a explicar el impacto diferencial de la pandemia entre los pueblos indígenas 
y el resto de la población nacional (Meneses-Navarro et al., 2020; Argoty-Pantoja et al., 2021). Probablemente esto puede ser explicado por las mayores barreras que enfrentan para el acceso a servicios de atención hospitalaria de calidad, sumado a la alta prevalencia de comorbilidades como diabetes, hipertensión arterial y enfermedad crónica renal (Ibarra-Nava et al., 2021).

El sureste de México concentra gran parte de la población indígena del país. La mayoría vive en comunidades rurales o urbano marginales en condiciones de pobreza y rezago educativo (Díaz de León-Martínez et al., 2020). Esto hace necesario implementar intervenciones sanitarias que atiendan tanto las desigualdades estructurales como las diferencias culturales y lingüísticas de los pueblos indígenas (Aranda et al., 2021). Este estudio analiza las percepciones de riesgo, emociones y comportamientos de jóvenes estudiantes residentes de regiones rurales e indígenas de gran marginación en el estado de Chiapas, México, sobre el COVID-19. Los resultados son útiles para formular recomendaciones de carácter intercultural que pueden contribuir a mejorar la respuesta del sistema de salud frente al COVID-19 y otras pandemias en el ámbito indígena y rural de México, y otros países de América Latina.

\section{MATERIALES Y MÉTODOS}

\section{Sitio de estudio}

En junio de 2020 se aplicó una encuesta a jóvenes estudiantes de entre 15 y 18 años pertenecientes al Colegio de Bachilleres de Chiapas (COBACH) que cursan estudios de preparatoria. Se eligieron 20 planteles ubicados en 17 municipios indígenas y rurales de alta y muy alta marginalidad de la entidad según el Consejo Nacional de Evaluación de la Política de Desarrollo Social (CONEVAL). Los planteles fueron seleccionados a través de un generador de números aleatorios utilizando el Software SPSS Statistics versión 15.0 (Figura 1). 
Figura 1. Ubicación de los sitios de estudio en zonas marginadas del estado de Chiapas, México

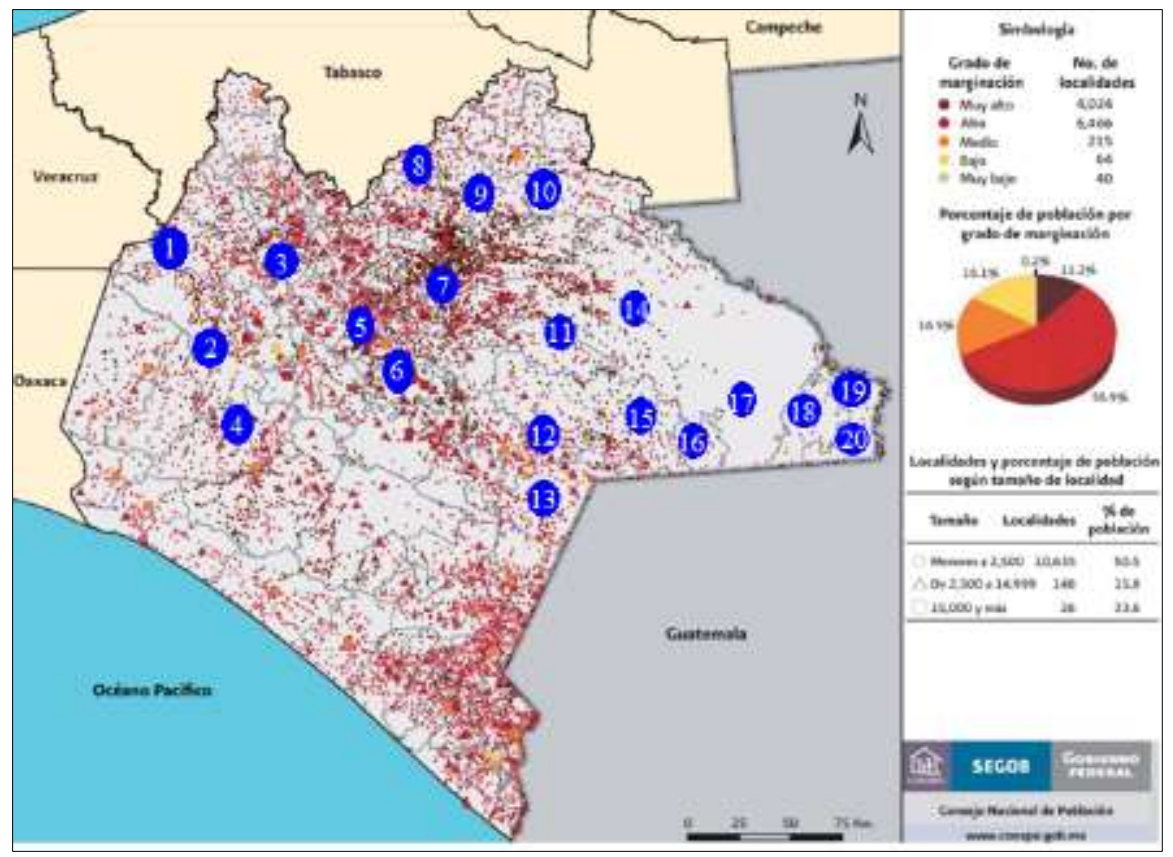

\section{Instrumento de investigación y aplicación}

La encuesta se diseñó con preguntas cerradas y abiertas que exploró aspectos como: a) el perfil socioeconómico de los estudiantes; b) los conocimientos sobre el origen y consecuencias del COVID-19; c) las percepciones de riesgo; d) las medidas de prevención familiares y comunitarias, e) las emociones respecto a la pandemia y f) los medios de comunicación e información que utilizan para mantenerse informados sobre la enfermedad. La encuesta se aplicó a 50 estudiantes de cada campus escolar, lo que resultó en una población de estudio de 1,000 participantes. Los estudiantes fueron seleccionados al azar de las listas oficiales de estudiantes matriculados, que fue el único criterio de inclusión.

\section{Análisis de los datos}

Los datos obtenidos de las encuestas fueron introducidos al software Epi Info versión 7.2 para su manejo y análisis descriptivo para calcular frecuencias y proporciones. Para evaluar diferencias entre grupos, se utilizó el software SPSS Statistics versión 15.0 para aplicar las pruebas U de Mann-Whitney y de Kruskal-Wallis para comparar dos o más de dos grupos. El valor de significancia estadística se estableció cuando $\mathrm{p} \leqslant 0.05$. 


\section{Consideraciones éticas}

En estudio se apegó a los lineamientos establecidos la Declaración de Helsinki (World Medical Association, 2013) y el Reglamento de la Ley General de Salud de México en materia de investigación para la salud (Secretaría de Gobernación. 2014). Antes de iniciar el estudio, se obtuvo una carta de consentimiento informado del comité de padres de cada establecimiento educativo, describiendo el objetivo y enfoque del proyecto. Para la protección de datos de los participantes, no se solicitaron nombres u otra identificación personal. La participación de los alumnos encuestados fue voluntaria, con la opción de rechazar la participación en cualquier momento.

\section{RESULTADOS}

\section{Perfil demográfico}

971 jóvenes respondieron la encuesta, $43.2 \%$ fueron hombres y 56.8\% mujeres; $93 \%$ de los estudiantes que participaron en el estudio tenían entre 15 y 18 años. Además de hablar español, $47.6 \%$ de los estudiantes fueron hablantes de alguna lengua indígena (Tabla 1).

Tabla 1. Perfil sociodemográfico de la población de estudio

\begin{tabular}{lcc}
\hline Sexo & Frecuencia & Porcentaje \\
Mujer & 413 & $43.20 \%$ \\
Hombre & 543 & $56.80 \%$ \\
\hline Edad & & \\
$15-17$ & 703 & $72.48 \%$ \\
$18-20$ & 262 & $27.01 \%$ \\
$21-22$ & 5 & $0.52 \%$ \\
\hline Habla lengua indígena & & \\
Sí & 442 & $47.63 \%$ \\
No & 486 & $52.37 \%$ \\
\hline
\end{tabular}

\section{Conocimiento sobre el origen y consecuencias del COVID-19}

92.2\% de los estudiantes señaló conocer o haber escuchado sobre el virus SARS-CoV-2 o la enfermedad conocida como COVID-19 y el $88.4 \%$ cree en la existencia de la enfermedad. Sin embargo, el 3.8\% de los encuestados se mantenían incrédulos respecto 
al virus y la enfermedad. Respecto a su origen, el 39.2\% cree que el virus fue creado en un laboratorio, el $26.9 \%$ opina que fue hecho por el gobierno y el $23.8 \%$ considera que su origen fue natural (Tabla 2).

\section{Percepciones de riesgo}

En junio de 2020, solamente $7.3 \%$ de los estudiantes encuestados conocía a alguna persona que hubiera padecido COVID-19; el 5.5\% a alguien que hubiera fallecido a causa de esta enfermedad y el $1.5 \%$ de los encuestados habían estado enfermos o convivido con alguna persona enferma por el nuevo coronavirus. El 14.2\% consideraba que podría contagiarse, aunque el $76.4 \%$ señaló sentirse amenazado o muy amenazado por esta enfermedad. El 84.4\% indicó que el COVID-19 representa una amenaza real para la salud y la vida de otros seres queridos; el $72.9 \%$ que es también una amenaza para la situación económica personal y de su familia, y el $70.8 \%$ que es una amenaza para la situación económica en su comunidad (Tabla 2).

\section{Gravedad percibida}

El 85.4\% de los jóvenes encuestados consideraron que la pandemia de COVID-19 era un asunto grave o muy grave en México, pero sólo 29.9\% consideraron que la situación era grave o muy grave en su comunidad. Al mismo, $46.1 \%$ opinaron que la pandemia era poco o nada grave en su comunidad (Tabla 2).

\section{Consecuencias en educación e ingresos}

El $87.7 \%$ de los encuestados habían suspendido clases escolares a causa de la pandemia y el 36\% había sufrido la reducción de los ingresos familiares en sus hogares por motivo del COVID-19. Para continuar con las actividades escolares no presenciales, sólo el $33.8 \%$ de los estudiantes pudieron tomar clases por internet y el $50.5 \%$ decidieron estudiar por su cuenta (Tabla 2).

\section{Medios de información}

El medio de información más utilizado para informarse sobre el avance de la pandemia de COVID-19 fue la televisión (62.4\%) seguido por las redes sociales $(29.1 \%)$ (Tabla 2). 
Tabla 2. Preguntas y respuestas del cuestionario sobre percepciones de riesgo al COVID-19 entre alumnos del Colegio de Bachilleres, Chiapas, 2020

\begin{tabular}{|c|c|c|c|c|c|}
\hline \multicolumn{2}{|r|}{ Preguntas } & \multirow{2}{*}{$\frac{\text { Sí }}{874}$} & \multirow{2}{*}{$\frac{\%}{92.2}$} & \multirow{2}{*}{$\begin{array}{c}\text { No } \\
73\end{array}$} & \multirow{2}{*}{$\begin{array}{c}\% \\
7.1\end{array}$} \\
\hline 1 & $\begin{array}{l}\text { ¿Conoces o has escuchado sobre el Coronavirus SARS-CoV2 o de la } \\
\text { enfermedad COVID-19? }\end{array}$ & & & & \\
\hline 2 & $\begin{array}{l}\text { ¿Crees que exista la enfermedad del COVID-19 o el virus que la } \\
\text { provoca SARS-CoV2? }\end{array}$ & 842 & 88.4 & 110 & 11.5 \\
\hline 3 & ¿Conoces personalmente a alguien que haya enfermado de COVID-19? & 71 & 7.3 & 890 & 92.6 \\
\hline 4 & ¿Conoces personalmente a alguien que haya fallecido de COVID-19? & 53 & 5.5 & 902 & 94.4 \\
\hline & $\begin{array}{l}\text { ¿Has convivido personalmente con alguna persona enferma de } \\
\text { COVID-19? }\end{array}$ & 14 & 1.5 & 915 & 98.4 \\
\hline 6 & ¿Estas o has estado enfermo de COVID-19? & 14 & 1.5 & 892 & 98.4 \\
\hline 7 & ¿Consideras que podrías contagiarte? & 125 & 14.2 & 126 & 14.3 \\
\hline \multirow[t]{3}{*}{8} & $\begin{array}{l}\text { ¿A causa del COVID-19 se han visto disminuidos los ingresos de tu } \\
\text { hogar? } \\
\text { ¿Qué emociones has experimentado a raíz de la epidemia por COVID- } \\
\text { 19?: }\end{array}$ & 323 & 36 & 408 & 45.4 \\
\hline & Tristeza & 164 & 16.8 & 807 & 83.1 \\
\hline & Enojo & 64 & 6.5 & 907 & 93.4 \\
\hline \multirow[t]{5}{*}{9} & Incertidumbre por lo que vaya a pasar & 111 & 11.4 & 860 & 88.5 \\
\hline & Preocupación & 428 & 44 & 543 & 55.9 \\
\hline & Miedo por mi salud y mi vida & 211 & 21.7 & 760 & 78.2 \\
\hline & Miedo por la salud y la vida de otros seres queridos & 444 & 45.7 & 527 & 54.2 \\
\hline & Incredulidad & 37 & 3.8 & 934 & 96.1 \\
\hline \multirow[t]{3}{*}{10} & $\begin{array}{l}\text { ¿Cómo consecuencia del COVID-19 se suspendieron las } \\
\text { presenciales? }\end{array}$ & 801 & 87.8 & 50 & 5.4 \\
\hline & ¿Qué estrategias has implementado para seguir aprendiendo?: & 306 & 31.5 & 665 & 68.4 \\
\hline & Estudiar por mi cuenta & 491 & 50.5 & 480 & 49.4 \\
\hline \multirow[t]{3}{*}{11} & He tomado clases en línea & 329 & 33.8 & 642 & 66.1 \\
\hline & He recibido guías de aprendizaje para estudiar en casa & 55 & 5.6 & 916 & 94.3 \\
\hline & $\begin{array}{l}\text { Me he reunido con otros compañeros para reunir inforn } \\
\text { resolver dudas }\end{array}$ & 306 & 31.5 & 665 & 68.4 \\
\hline
\end{tabular}

¿Cuál crees que es el origen del Coronavirus COVID 19?

Frecuencia $\quad \%$

Fue creado por la naturaleza

$218 \quad 23.8$

12 Fue creado en laboratorio $\quad 359 \quad 39.2$

Lo creó el gobierno $\quad 246 \quad 26.9$

Otro origen

$91 \quad 9.9$

\begin{tabular}{|c|c|c|c|c|c|c|c|c|}
\hline & \multicolumn{2}{|c|}{$\begin{array}{c}\text { Muy } \\
\text { amenazado }\end{array}$} & \multicolumn{2}{|c|}{ Amenazado } & \multicolumn{2}{|c|}{$\begin{array}{c}\text { Poco } \\
\text { amenazado }\end{array}$} & \multicolumn{2}{|c|}{$\begin{array}{c}\text { No } \\
\text { amenazado }\end{array}$} \\
\hline & $\mathbf{F}$ & $\%$ & $\mathbf{F}$ & $\%$ & $\mathbf{F}$ & $\%$ & $\mathbf{F}$ & $\%$ \\
\hline $\begin{array}{l}13 \text { ¿Crees que el COVID-19 es una amenaza } \\
\text { para tu vida? }\end{array}$ & 359 & 39.6 & 319 & 35.8 & 164 & 18.2 & 60 & 6.6 \\
\hline $\begin{array}{l}14 \text { ¿Crees que el COVID-19 es una amenaza } \\
\text { para la salud y vida de tus seres queridos? }\end{array}$ & 391 & 43.1 & 375 & 41.3 & 107 & 11.8 & 33 & 3.6 \\
\hline
\end{tabular}


¿Crees que el COVID-19 es una amenaza

15 para tu situación económica y la de tu 355 $39.4302 \quad 33$ familia?

¿Crees que el COVID-19 es una amenaza

16 para la situación económica de tu

$\begin{array}{llllllll}334 & 37.1 & 303 & 33.7 & 185 & 20.6 & 76 & 8.4\end{array}$ comunidad?

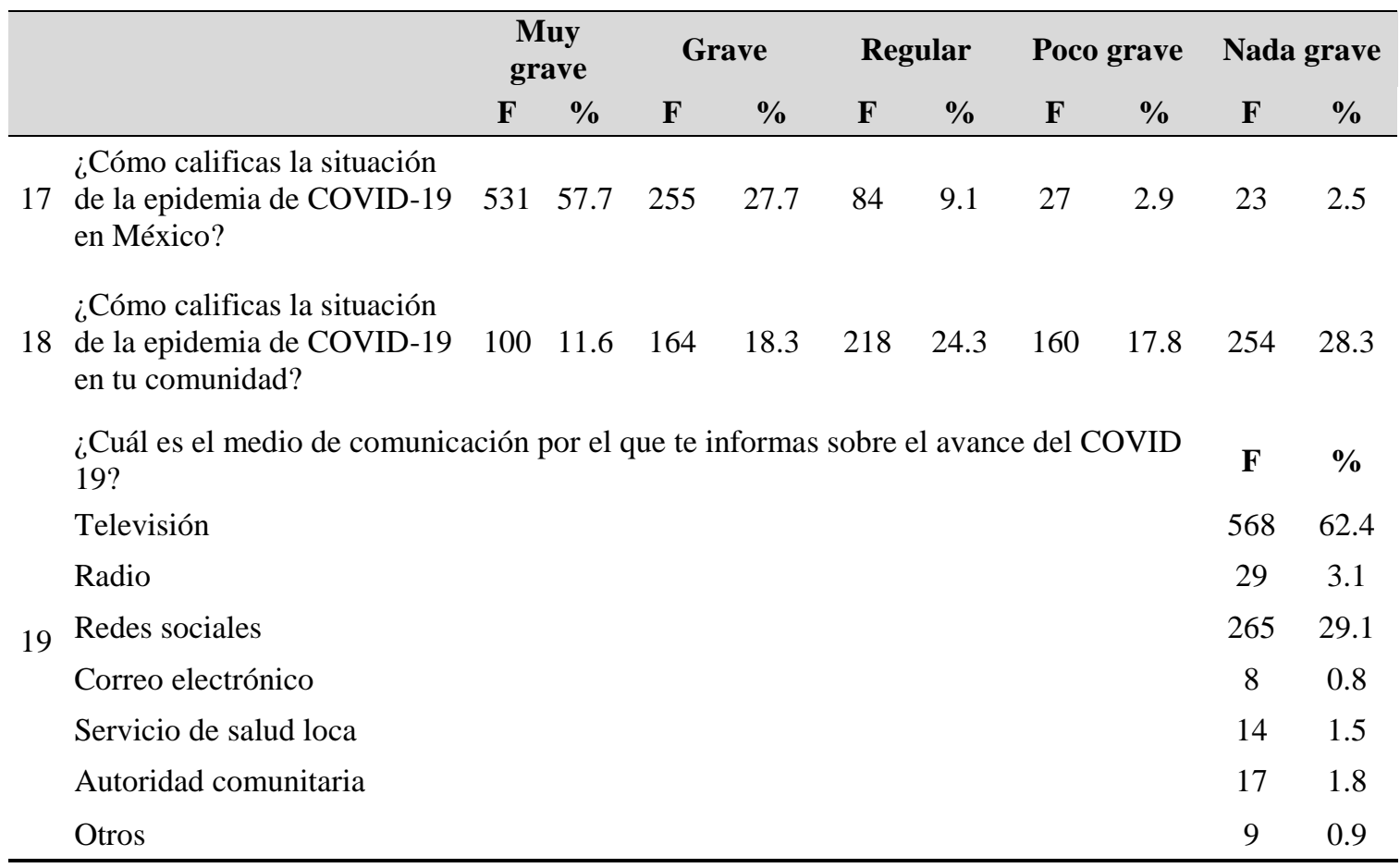

\section{DISCUSIÓN Y CONSIDERACIONES FINALES}

Aunque poco menos de la mitad de los estudiantes encuestados son hablantes de alguna lengua indígena, podemos suponer que la mayoría se auto adscriben como indígenas o pertenecen a familias donde alguno de los padres es indígena, toda vez que los planteles del Colegio de Bachilleres de Chiapas (COBACH) se ubican en municipios predominantemente indígenas del estado. Esto permite aseverar que nuestro análisis brinda un panorama general de las percepciones de riesgo frente al COVID-19 de jóvenes indígenas de comunidades rurales de gran marginación.

Es de destacar que, a pesar de la histórica desconfianza e incredulidad de las poblaciones indígenas hacia las autoridades sanitarias, la amplia mayoría de los encuestados (92.2\%) creen en la existencia del virus SARS-CoV2 y del COVID-19 (Murillo, 2020). Sin embargo, este dato contrasta con las muestras de rechazo hacia las acciones sanitarias. En diversas regiones del estado se produjeron expresiones violentas como la destrucción de centro de salud y ambulancias, o las agresiones al personal de 
salud que realizaba actividades de "sanitización" o búsqueda de casos de enfermos de COVID-19 (Mariscal, 2020). Estas manifestaciones de desconfianza y rechazo hacia las medidas de respuesta ante la pandemia también se presentaron en comunidades indígenas de otras entidades del país ${ }^{19}$. En algunas ocasiones, el argumento para estas agresiones se basaba en la idea de que las propias autoridades de salud y gubernamentales eran las responsables de provocar y esparcir la enfermedad ${ }^{6}$. Esto concuerda con el hallazgo de que un porcentaje significativo de los estudiantes encuestados cree que el virus fue creado en algún laboratorio y/o con la participación del propio gobierno, con malas intenciones dirigidas específicamente a grupos sociales subalternos. Estas ideas coinciden en general con diversas teorías conspirativas que se han difundido ampliamente a través de internet -particularmente en las redes socialesrespecto a la pandemia del coronavirus SARS-CoV2 (20), y que comparten los contenidos esenciales de otras teorías conspiracionistas (Vega-Dienstmaier, 2020) que se trata de una enfermedad creada, que es un complot de la industria farmacéutica, que es un pretexto para insertar nanotecnologías mediante las vacunas para el control de la población) que se han presentado en otras pandemias recientes previas como las de Zika y Chikungunya (Zúñiga, 2016).

Debe ser de particular preocupación los efectos de la incredulidad y la desconfianza entre las poblaciones indígenas sobre la vacunación contra el COVID-19 (Aranda et al., 2021). Particularmente en Chiapas, diversas comunidades y municipios enteros han expresado su renuencia o rechazo explícito hacia la vacunación contra COVID-19 e, incluso, contra el esquema completo de vacunación para niños y adultos (AFP, 2021, Mariscal, 2021). Del mismo modo, consideran que las vacunas tienen un propósito negativo para la sociedad, desde que la vacuna contiene al agente causal o que estas sirven para marcar, vigilar y controlar a los individuos. Esta renuencia a las campañas de vacunación puede retrasar el retorno a las actividades normales de toda índole, con sus consecuencias perniciosas tanto para la salud de las poblaciones como las economías.

Estos hallazgos apuntan a la imperiosa necesidad de incrementar las estrategias de comunicación enfocada a la población juvenil indígena que privilegie información validada científicamente y que desestime teorías conspiratorias y falsas en lo relacionado con el origen del virus. Cabría esperar que, toda vez que los alumnos de 
nivel preparatoria del COBACH representan a uno de los sectores más cercanos al conocimiento y pensamiento científico en el ámbito indígena y rural, precisamente ellos fungieran como agentes para contrarrestar la desinformación y contribuir a la difusión de información basada en evidencia, de manera clara, con pertinencia cultural y en el idioma predominante en sus comunidades.

Cabe señalar que en el periodo de aplicación de la encuesta (junio de 2020), en México se cumplían dos meses del aislamiento domiciliario, mismo que fue indicado también en las comunidades rurales, aunque el avance de la enfermedad era aún incipiente en las localidades más alejadas del país (INSP, 2020). La baja experiencia directa de la enfermedad entre los encuestados, sea que hubieran enfermado o conocieran a alguien que padeciera la enfermedad o hubiera fallecido por su causa, puede explicarse debido a que al momento de la encuesta la epidemia se concentraba en las principales zonas urbanas del país y apenas iniciaba su difusión al ámbito rural. A más de un año de distancia, es probable que la experiencia directa de la enfermedad sea mayor.

No obstante, la amplia mayoría consideraron que el COVID-19 es una amenaza real para ellos y para la salud, la vida y la economía de sus familiares y comunidades. Precisamente por las mismas fechas en que se aplicó esta encuesta, en diversas comunidades y regiones indígenas del país se implementaron medidas de cierre de los caminos de acceso que impidieron la entrada o la salida a las mismas (Palomares, 2020; Martínez-Elorriaga, 2020; Llaven-Anzures, 2020; Cancino, 2020; García, 2020), lo que propició diversos inconvenientes para el bienestar y la economía locales: dificultades para el abasto de alimentos y utensilios domésticos o de labranza, el impedimento para llevar a cabo actividades laborales fuera de la comunidad, el agravamiento de las condiciones de pobreza preexistentes, las dificultades para el acceso a los servicios de salud, entre otros.

De manera temprana, la pandemia de COVID-19 acarreó efectos negativos en otros ámbitos del bienestar social: casi nueve de cada diez encuestados suspendieron las clases escolares por causa de la contingencia y sólo una tercera parte pudieron continuar con su formación escolar a través de medios virtuales; del mismo modo, la tercera parte de los hogares registraron reducciones en sus de por sí bajos ingresos monetarios.

Los resultados de este estudio reflejan que los jóvenes bachilleres mostraron desconocimiento e imprecisiones sobre el origen del virus, y desconfianza hacia las 
autoridades de salud y las medidas contra la pandemia. Por esta razón se requiere implementar programas de comunicación y de difusión en materia de salud que incrementen el conocimiento sobre el origen del virus SARS-CoV-2 y genere entornos de confianza hacia las instancias de gobierno responsables del control de la pandemia. Esto favorecerá la apropiación masiva de las medidas de protección en las comunidades indígenas, incluyendo la vacunación, misma que se ha visto limitada por la desinformación y la desconfianza (Rios-González, 2021).

\section{REFERENCIAS BIBLIOGRAFICAS}

AFP, . San Juan Cancuc, un poblado indígena de Chiapas que rechaza vacunarse contra el COVID. Animal Político. Disponible en: https://www.animalpolitico.com/2021/02/san-juan-cancuc-chiapas-rechazavacuna-covid/

Mariscal, A. (2021). Mejor que me recoja Dios y no el kaxlan. 2021; Chiapas Paralelo, Disponible en: https://www.ladobe.com.mx/2021/07/mejor-que-me-recoja-diosy-no-elkaxlan-el-que-viene-de-fuera/

Aranda, Z., Arana-Cedeño, M., Meléndez-Navarro, M., Meneses-Navarro, S., y Sánchez-Pérez, H.J. (2021). A call for COVID-19 immunization campaigns that address the specific circumstances of indigenous peoples of Latina America and the Caribbean. Lancet Regional Health Americas, 3, doi: 10.1016/j.lana.2021.100074.

Argoty-Pantoja, A.D,. Robles-Rivera, K., Rivera-Paredez, B., Salmerón, J. (2021). COVID-19 fatality in Mexico's indigenous populations. Public Health, 193:6975. https://doi.org/10.1016/j.puhe.2021.01.023

Cancino, K. (2020). Se aíslan las zonas indígenas en Nayarit para evitar contagios”. El Heraldo de México. Disponible en: https://heraldodemexico.com.mx/nacional/2020/4/6/se-aislan-las-zonasindigenas-en-nayarit-para-evitar-contagios-165611.html

De Fuentes-Vicente, J.A., Hernández-Reyes, N., Bermúdez-Urbina, F.M., MenesesNavarro, S., Castillo-Hernández, A.L., Santos-Hernández, N.G., GutiérrezJiménez, J., y Córdova-Aguilar A. (2021). Knowledge and perceptions about COVID-19 in the marginalized population of southeastern Mexico. Salud Pública de México, 63(5):589. https://doi.org/10.21149/12547 
Díaz de León-Martínez, L., de la Sierra-de la Vega, L., Palacios-Ramírez, A., Rodriguez-Aguilar, M., y Flores-Ramírez, R. (2020). Critical review of social, environmental and health risk factors in the Mexican indigenous population and their capacity to respond to the COVID-19. Science of the Total Environment, 733, 139357. doi: 10.1016/j.scitotenv.2020.139357.

García, J. (2020). Los indígenas de México se cierran para frenar al coronavirus”. El País (España). Disponible en: https://elpais.com/sociedad/2020-04-21/losindigenas-de-mexico-se-cierran-para-frenar-alcoronavirus.html\#: :text=El\%20sistema\%20de\%20'usos\%20y\%20costumbres' $\% 20$ es\%20un\%20modelo\%20de,de\%20poblaci\%C3\%B3n\%20ind\%C3\%ADgen a\%20de\%20M\%C3\%A9xico.\&text=Ah\%C3\%AD\%20las\%20autoridades\%20de fienden $\% 20$ que,a\%20que $\% 20$ no $\% 20$ hay $\% 20$ hospitales.

Haro, J. (2020). Dossier: pueblos indígenas mexicanos frente a la pandemia COVID-19. México: El Colegio de Sonora. Disponible en: https://www.clacso.org/wpcontent/uploads/2020/05/Haro-2020-Dossier-Indigenas-mexicanos-frente-a-lapandemia-Covid19.pdf

Ibarra-Nava, I., Flores-Rodríguez, K., Ruiz-Herrera, V., Ochoa-Bayona, H., SalinasZartuche, A., Padilla-Orozco, M., y Salazar-Montalvo, R. (2021). Ethnic disparities in COVID-19 mortality in Mexico: A cross-sectional study based on national data. PLoS One, 16(3):e0239168.

Instituto Nacional de Salud Pública. (2020). Reflexiones sobre la respuesta de México ante la pandemia de COVID-19 y sugerencias para enfrentar los próximos retos. Nota conceptual. México: Instituto Nacional de Salud. Disponible en: https://www.insp.mx/avisos/recomendaciones-pandemia

Llaven-Anzures, Y. (2020). Autoridades de la Sierra Norte y Mixteca prohíben ingreso de foráneos y migrantes hasta que concluya la emergencia sanitaria”. La Jornada de

Oriente. Disponible

en: https://www.lajornadadeoriente.com.mx/puebla/autoridades-de-la-sierra-norte-ymixteca-prohiben-ingreso-de-foraneos-y-migrantes-hasta-que-concluya-laemergencia-sanitaria/

Martínez-Elorriaga, E. (2020). Instalan cerco sanitario en comunidad purépecha en Michoacán”. La Jornada. Disponible en: 
https://www.jornada.com.mx/ultimas/estados/2020/04/05/instalan-cercosanitario-en-comunidad-purepecha-en-michoacan-7609.html

Meneses-Navarro, S., Freyermuth-Enciso, M.G., Pelcastre-Villafuerte, B., y GómezFlores-Ramos, L. (2021). COVID-19 among indigenous populations and the response of health system in Mexico" en Nicholas Spence, COVID-19 \& indigenous peoples in the world, University of Toronto. En prensa

Meneses-Navarro, S., Freyermuth-Enciso, M.G, Pelcastre-Villafuerte, B.E., CamposNavarro, R., Meléndez-Navarro, D., y Gómez-Flores-Ramos, L. (2020). The challenges facing indigenous communities in Latin America as they confront the COVID-19 pandemic. International Journal of Equity and Health, 19(63). https://doi.org/10.1186/s12939-020-01178-4

Miranda, F. (2020) Coronavirus: Celebra comunidad mixe de Oaxaca fiesta religiosa multitudinaria, El Universal. Disponible en: https://www.eluniversal.com.mx/estados/coronavirus-celebra-comunidad-mixede-oaxaca-fiesta-religiosa-multitudinaria.

Murillo, A. (2021). Desconfianza, regímenes de verdad, conspiraciones y montajes en el contexto de la covid-19 en México. Desde el Sur, 12(2): 547-571. http://dx.doi.org/10.21142/des-1202-2020-0030

Palomares, J. (2020). Ante amenaza de Covid-19, cierran entrada a territorio de la etnia guarijío". El Imparcial (Hermosillo) Disponible en: https://www.elimparcial.com/sonora/sonora/Ante-amenazada-de-Covid-19cierran-entrada-a-territorio-de-la-etnia-Guarijio--20200324-0025.html

Pelcastre-Villafuerte, B.E., Meneses-Navarro, S., Reyes-Morales, H., y RiveraDommarco, J.A. La salud de los pueblos indígenas: retos y perspectivas. Salud Publica de México, 62(3):221-222. https://doi.org/10.21149/10785

Pelcastre-Villafuerte, B.E., Meneses-Navarro, S., Sánchez-Domínguez, M., MeléndezNavarro, D., y Freyermuth-Enciso G. (2020). Condiciones de salud y uso de servicios en pueblos indígenas de México. Salud Pública de México, 62:810819. https://doi.org/10.21149/11861

Rios-González, C.M. (2021). Predisposición para recibir la vacuna contra el COVID 19 en Paraguay: estudio exploratorio. Medicina Clínica y Social, 5(2): https://doi.org/10.52379/mcs.v5i2.189 
Sánchez-Talanquer, M., González-Pier, E., Sepúlveda, J., Abasca-Miguel, L., Fieldhouse, J., Del Rio, C., y Gallalee, S. (2021). Mexico's Response to COVID-19: A Case Study. San Francisco. Disponible en: https://globalhealthsciences.ucsf.edu/sites/globalhealthsciences.ucsf.edu/files/me xico-covid-19-case-study-english.pdf

Secretaría de Gobernación. (2014). Reglamento de la ley general de salud en materia de investigación para la salud. Ciudad de México. Disponible en: http://www.diputados.gob.mx/LeyesBiblio/regley/Reg_LGS_MIS.pdf

Suárez, V., Suarez-Quezada, M., Oros-Ruiz, S., y Ronquillo De Jesús, E. (2020). Epidemiology of COVID-19 in Mexico: From the 27th of February to the 30th of April 2020. Revista Clínica Española, 220(8):463-471. doi: 10.1016/j.rce.2020.05.007

Vega-Dienstmaier, J. (2020). Teorías de conspiración y desinformación en torno a la pandemia de la COVID-19. Revista de Neuropsiquiatría, 83(3):135-137. https://doi.org/10.20453/rnp.v83i3.3792

World Medical Association. (2013). World Medical Association Declaration of Helsinki: ethical principles for medical research involving human subjects. JAMA, 310(20):2191-4. doi: 10.1001/jama.2013.281053.

Zúñiga, D. (2016). Cinco teorías conspirativas sobre el virus del Zika. DW, disponible en: https://www.dw.com/es/cinco-teor\%C3\%ADas-conspirativas-sobre-el-virusdel-zika/a-19030087 